\title{
Promotion of Self-Care in Clinical Practice: Implications for Clinical Supervision in Nursing
}

\author{
Wilson Abreu, Cristina Barroso, Maria de Fátima Segadães, and Silvia Teixeira
}

\begin{abstract}
Self-care is a human function that should be carried out by the individuals themselves, in order to preserve their life, health and self-esteem. During the past ten years, some approaches used by physicians and nurses to support self-care were identified to complement professional practices and structures, such as clinical supervision or leadership. This quantitative study was developed in a hospital in the North of Portugal. The start question was: "How to organize the system of clinical supervision in order to promote self-care and to contribute to the quality of care?" Participants were 110 patients hospitalized in a ward of the above-mentioned hospital. Two instruments were used to collect data. The first was a questionnaire focused on self-care and was filled out by rehabilitation nurses. The second instrument evaluated self-care as recorded in nurses' electronic records filled during the caring process. Data analysis pointed out that self-care notes in patient anamnesis were limited to hygiene, while the degree of dependence was often ignored. There appears to be some confusion in the records regarding concepts and evaluation of the degree of dependence in self-care. Suggestions are made to promote self-care inside the system of clinical supervision, in order to provide appropriate healthcare to patients.
\end{abstract}

Index Terms-Clinical supervision, self-care, dependency, quality of care.

\section{INTRODUCTION}

Dependency is defined as the incapacity of a person to function satisfactorily without the help of equipment or of other people that enable them to adapt. The inability of any individual to take care of themselves suggests that the person requires aid in their daily activities that they are not able to carry out because they either cannot or do not wish to. They therefore require aid in order to survive [1]. Dependency refers to a situation in which individuals, for reasons that maybe internal or external to the person, cannot carry out their daily activities on their own.

According to Orem [2], illness may limit what people can accomplish on their own, to the extent that their thinking, decision making and initiative for self-care activities are altered. Orem [3] also highlighted that healthy adults are able to take care of themselves but, due to illness or physical or psychological disturbances, or to a lack of resources or environmental factors, the individual need for self-care becomes greater than their capacity to carry it out, limiting

Manuscript received November 4, 2013; revised January 16, 2014.

Wilson Abreu, Cristina Barroso, and Maria de Fátima Segadães are with the Porto School of Nursing, Porto, Portugal. Rua Dr. António Bernardino de Almeida, CO 4200-072 Porto (e-mail: wjabreu@esenf.pt, cmpinto@esenf.pt fsegadaes@esenf.pt).

Silvia Teixeira is with the Porto School of Nursing, Porto, Portugal. Rua Dr. António Bernardino de Almeida, CO 4200-072 Porto (e-mail: silvia.smmt@gmail.com). what people can do for themselves. Self-care support can come from family members, neighbours, friends or health care professionals.

Self-care is a human regulatory function that should be carried out by the individuals themselves, or that may be performed for them in order to preserve their life, health and good self-esteem [4]. It is defined as those deliberate activities needed to maintain levels of health and well-being [5]. This means that it encompasses health care and illness prevention measures [6]-[8].

A paradigm shift is now recognized to have occurred. Self-care has assumed a central role in many disciplines, within the healthcare field and social work. This is a fact which suggests that the goal of any intervention has ceased to be the illness itself but has been replaced by the patients' response to the illness and to life processes, as well as the transitions experienced by individuals and their families throughout life's long cycle.

According to Schumacher [9], there are several different healthcare approaches. In the self-care process, the person takes care of themselves, with the caregivers playing merely a supportive rather than care giving role. According to a collaborative pattern, individuals take care of some basic daily needs but the caregiver has a more active role. In the substitutional pattern, the caregiver completely takes over the dependent person's daily activities.

Abreu and Morrow [10] state that clinical supervision (CS) is both a collaborative and a supportive process between two or more nurses to encourage the development of professional skills, promote quality standards in practice and enhance the quality and safety of patient care; they also maintain that CS is a central component of nursing culture and life on the wards. Hancox et al. [11] argue that CS is a tool for developing nursing care, basically because it directs attention to the development of skills, knowledge and professional values by way of increasing nurses' autonomy and enabling them to take responsibility for the health care provided. It sets up conditions for reflection about the practice and the defence not only of caregiver values and interests of the supervised caregiver but also those of the organization in which the supervision takes place [12]. However, Abreu and Morrow [10] point out that much of the research has illustrated that there are problems relating to a lack of understanding of the concept, poor training of clinical supervisors and a lack of support and commitment to the practice of clinical supervision.

If we take into consideration the need to promote the quality of healthcare, studying the effects of CS on the quality of care suggests a timely and useful object of study [13]. According to Cruz [14], CS has been proven to have positive effects at many different levels. Many studies have been carried out but few have concentrated on patients' health 
status evolution or on the quality of care [15].

This study is just one part of a larger research developed in the hospital, whose subject is the implementation of a system of CS in the wards. We have focused on self-care not only because we found it to be one of the indicators of quality of health care but also because it serves as a benchmark for measuring professional quality as it is a central concern to clinical practice. It is a healthcare outcome that is directly susceptible to nursing interventions with positive effects on health and personal well-being. Thus, taking into consideration the fact that one of the principal goals of CS is to promote the quality of care, we decided that self-care is one of the areas in which CS might have a positive effect.

\section{MethodolOGY}

\section{A. Research Question and Hypothesis}

According to Lee and Miller [16] self-care is widely recognized as critical to social work and health care, yet little empirical support or practical guidance exists in the literature to steer workers towards its implementation. Not only is self-care crucial in preventing secondary health problems, but it can also serve as a means of empowerment, enabling patients to proactively and intentionally negotiate their overall health, well-being and resilience.

This quantitative study took place in a medium sized hospital in the north of Portugal. The start research question was the following: "How to organize the system of clinical supervision in order to promote self-care and to contribute to the quality of care?" Each of the following guiding hypotheses focused on the situation and patients' clinical context were formulated:

$\mathrm{H} 1$ : There is a statistically significant relationship between the patient age and the degree of self-care dependence.

$\mathrm{H} 2$ : There is a statistically significant relationship between patient gender and the degree of self-care dependence.

H3: There is a statistically significant relationship between the medical diagnosis and the level of dependence in self-care.

H4: There is a statistically significant relationship between the self-care dependence at home and during hospitalization.

$\mathrm{H} 5$ : There is a statistically significant relationship between the degree of dependence on a specific self-care and those of the others.

H6: There is a statistically significant relationship between the degree of dependence identified by nursing specialists and the degree of independence identified by the generalists.

H7: There is a statistically significant relationship between the interventions prescribed by nursing specialists and those suggested by generalists.

\section{B. Sampling}

Participants were patients hospitalized in a ward of the above-mentioned hospital. A non-probabilistic, accidental sample $(N=110)$ was obtained of patients hospitalized during the research period between March 11th and April 24th, 2012.

\section{Data Collection and Analysis}

Two data-gathering instruments were used. The first was focused on self-care and filled out by rehabilitation nurses. It had three parts: a) patient data; b) self-care dependency evaluation; and c) the selection of nursing interventions according to the degree of dependence (DD) identified. The second instrument evaluated self-care as saved in nurses' records. It included the same three parts as the first but was filled by the researcher during an analysis of nursing records using SAPE® (electronic support system for nursing practice). The goal was to analyse the level of agreement between the data saved on the records by general nurses and the instrument applied by specialized rehabilitation nurses.

Statistical analysis of data was performed using SPSS ${ }^{\circledR}$ (version 19.0). Patient characterization data were analysed descriptively. A Qui-Squared (X2) test sought the level of association between categorical variables and Spearman correlation coefficient (rs) was used when interval variables with ordinals or ordinal-ordinal variables were found. A Wilcoxon (Z) test was used when data were measured on an interval scale. The level of significance for all tests was set at $\rho<0.05$.

\section{FINDINGS}

The sample included 42 women and 68 men aged between 25 and 95. Average age was 73.14. For both sexes, the most common age range was 65-79. Sixty-one participants (55.5\%) were dependent at home on some type of self-care, while the age range with the highest levels of dependence was the 80+ group. There was a wide degree of variation in the diagnoses, with the three most common being respiratory illnesses and disorders $(n=47 ; 42.7 \%)$, circulatory illnesses and disorders $(N=30 ; 27.3 \%)$ and disorders of the kidneys and urinary tract $(n=12 ; 10.9 \%)$.

The clinical history of $79.9 \%(n=78)$ of the participants included an initial evaluation of self-care, particularly as it referred to hygiene. Only 18 cases had a record of degree of dependence (DD), in six of which the note was made under the rubric of "motor activity" rather than "self-care". In one case, no explicit mention was made to the level of self-care, but a DD note was included.

The data obtained regarding the DD in self-care is as follows. (H1) A slight to moderate positive correlation was found between age and all types of self-care as indicated by specialist nurses (the higher the age, the greater the DD), except in "use of a wheelchair" in which the correlation was negative. The results from nursing records were similar except for the following forms of self-care: "getting dressed/ undressed", "personal grooming" and "standing up", in which no statistically significant correlation was observed. With the exceptions listed above, nursing records confirmed this hypothesis. Hypothesis (H2) that patient gender would correlate with nursing records was only confirmed in the dimension "changing positions". In general, women manifested greater DD then men in most types of self-care.

It was impossible to confirm (H3) from either source of data (specialist nurses or medical records) since in both cases there were more than $20 \%$ of cells with expected frequencies less than five in all forms of self-care. The minimum conditions for carrying out the statistical tests were not met. When the DD and the diagnosis at hospitalization were correlated, however, it was found that, with the exception of "independent eating", "turning oneself" and "using a 
wheelchair", that the diagnosis most closely related to the highest and middle range levels of DD was that of respiratory disorders.

Regarding (H4) that predicted a correlation between dependency at home and upon hospitalization, only the hypothesis as evaluated by the specialist nurses was subject to analysis, since in the medical records more than $20 \%$ of the cells has expected frequencies less than five.

(H5) included the relationship between different self-care behaviours and the view of specialists revealed a strong positive correlation between the DD of all types of self-cares except "using a wheelchair" and a slight to moderately negative correlation among the other types of self-care behaviours. It can be concluded that a statistically significant relationship exists between the DD of one type of self-care and that of other types. The hypothesis is, therefore, supported. In the medical records, however, all of the individual behaviours were confirmed except for the following "hygiene - dressing/undressing", "hygiene personal grooming", "personal grooming - hygiene (personal)", "standing - moving" and "standing - changing position." No significant correlations were found for these behaviours.

Hypothesis number six (H6) included the difference between specialist evaluations and medical records. The Wilcoxon test showed significant results for all self-care behaviours $(p<0.05)$ except "moving" $(p=0.265)$. Following the analysis of the relationships between the different types of self-care and the DD, it was concluded that no correlation was found between the specialists' evaluation of the DD and that recorded in the clinical records regarding the healthcare provided.

Specifically for those interventions related to equipment and adaptive strategies, it was found that specialist nurses regularly suggested interventions designed to teach, instruct and train patients while the medical records included no mention of such activity.

The test of hypothesis $\mathrm{H} 7$ found significant statistical differences $(p<0.05)$ between the interventions chosen by specialist nurses and those of the medical records; the hypothesis was, therefore, generally confirmed.

\section{DISCUSSION}

These results were important because some gaps were identified between nursing diagnoses and interventions in matter of self-care. Thus, they have identified opportunities for professional development in the self-care promotion related field. This study can contribute to the design of models of CS for improving nursing care. Data analysis pointed out that self-care notes in patient histories were limited to hygiene, while DD was often ignored. Statistically positive correlations were found (at significance levels lower than 0.05 ) between age and DD (the greater the age, the higher level of DD) and the existence of home DD (those patients with high levels of DD at home were also dependent during hospitalization). The DD of one type of self-care was statistically associated with all other DD behaviours. Significant statistical differences were found between the DD and the interventions identified both according to general nursing records and specialist evaluations.
In patients' clinical records, $79.9 \%$ of cases had references to self-care but only as it related to hygiene. The number is in fact very high. However, the cases without any mention of self-care are worrisome since the patient history brings together all of the information about the patient at the time of hospitalization and provides the basis for the nursing team to better and more uniformly plan healthcare throughout hospitalization. Thus, it would be expected that the history include all the relevant information. DD in self-care was often neglected, appearing in only $16.4 \%(n=18)$ cases. Of these 18 patients, the DD was sometimes noted not in the self-care field but in reference to motor activity.

The degree of dependence in self-care is a highly important and defining component of nursing interventions. The data reflected highly frequency of high levels of dependence in almost all aspects of self-care. Nursing records were more likely than specialist nurses to register cases of "no dependency". There was a total lack of correlation in all types of self-care between the two sources of data.

There appeared to be some confusion in the records regarding the fields of "walking", "self-care in dressing" and "dressing/undressing", suggesting the need for further study and improved operationalization of these terms within nursing teams. There is an obvious need for clarification of the concepts used in order to provide healthcare that is appropriate for actual patient needs. All of the specialist nurses marked the intervention area "evaluate", while none of the nursing records did so, since it was not provided on the SAPE ®form. This does not mean that nurses don't evaluate the interventions.

Statistically significant differences were found in the interventions within the two data sources for all types of self-care. Substitution interventions are those most regularly recorded as they are those in common between the specialist' evaluations and the nursing records. It should be noted that there is a need for critical reflection about the planning of care and its targeting towards patients' actual needs. A few notes should be made based on the data gathered from the case records: a) in some cases there were diagnoses made without subsequent interventions; b) there were cases in which interventions were recorded without any accompanying diagnosis; and c) no interventions were recorded targeting teaching, instruction or training, which raises the question of whether they did not exist or whether they took place but were not recorded.

Several shortcomings were identified in the nursing process. In order to address them, some potential self-care promotion strategies are suggested for inclusion in the CS model: setting up a team of nurses specialized in the promotion of self-care who could run simulations and provide group supervision; training in the mental skills and thought processes necessary for establishing procedures for techniques of nursing diagnoses. Occasional additional individual training sessions might be needed to fulfil specific needs not met with group supervision. Finally, self-care intervention protocols should be set up based on available scientific evidence.

\section{CONCLUSION}

According to Slusher, Withrow-Fletcher and 
Hauser-Whitaker [17], active involvement in self-care is widely acknowledged as an important strategy for achieving national health goals. An understanding of the underlying theoretical perspectives and paradigms embedded within acute and chronic disease management will facilitate nurses' engagement in the debate, practice within appropriate ethical boundaries and support individuals, families and communities more effectively in managing chronic disease [18]. Overall, the quality of healthcare provided by nurses depends on their ability to have an impact on self-care. Thus, none of the processes related to clinical intervention (CS, clinic leadership and clinical governance) can afford to ignore the concept and practice of self-care promotion. It is undeniable that the best nursing intervention requires reflective practice that can be enhanced with CS [10], [19]. Moreover, "since the effect of nurses' decision about their patients has such an enormous impact... it would be a grave mistake to neglect or hide the positive effects of clinical supervision" [14].

\section{REFERENCES}

[1] S. Pavarini and A. Neri, "Compreendendo dependência, independência e autonomia no contexto domiciliar: conceitos, atitude e comportamentos," in Atendimento Domiciliar: Um Enfoque Gerontológico, Y. Duarte and M. Diogo (eds.), São Paulo: Atheneu, 2000, pp. 49-70.

[2] D. Orem, Nursing: Concepts of Practice, 6 ed., St. Louis, MO: Mosby, 2001, p. 46.

[3] D. Orem, Nursing Theories and Nursing Practice, M. E. Parker, Ed., Philadelphia: Davis Co., 2005, p. 46.

[4] S. Richards, "Self-care in practice," Practice Nurse, vol. 37, no. 11, pp. 26-28, June 2009.

[5] F. Raffi, F. Shahpoorian, and M. Azarbaad, "The reality of learning self-care needs during hospitalization: Patients' and nurses' perceptions," Self-Care, Dependent-Care \& Nursing, vol. 16, no. 2, pp. 34-39, Oct. 2008.

[6] A. Richard and K. Shea, "Delineation of self-care and associated concepts," Journal Nursing Scholarship, vol. 43, pp. 255-264, Sep. 2011.

[7] K. Backman and M. Hentinen, "Factors associated with the self-care of home-dwelling elderly," Scandinavian Journal of Caring Sciences, vol. 15, no. 3, pp. 195-202, Sep. 2001.

[8] K. Backman and M. Hentinen, "Model for the self-care of home-dwelling elderly," Journal of Advanced Nursing, vol. 30, no. 3, pp. 564-572, Sep. 1999.

[9] L. Schumacher, "Reconceptualising family caregiving: Family-based illness care during chemotherapy," Research in Nursing \& Health, vol. 19, pp. 261-271, Aug. 1996.

[10] W. Abreu and C. Marrow, "Clinical supervision in nursing practice: A comparative study in Portugal and the United Kingdom," SANARE Políticas Públicas, vol. 11, no. 2, Dec. 2013.

[11] K. Hancox, L. Lynch, B. Happell, and S. Biondo, "An evaluation of an educational program for clinical supervision," International Journal of Mental Health Nursing, vol. 13, pp. 198-203, Sep. 2004.

[12] O. dos Enfermeiros, "Caderno temático Modelo de desenvolvimento profissional. Fundamentos, processos e instrumentos para a operacionalização do sistema de certificação de competências," Lisboa, PT: OE-Conselho de Enfermagem, 2010.

[13] K. Hyrkäs and K. Lehti, "Continuous quality improvement through team supervision supported by continuous self-monitoring of work and systematic patient feedback," Journal of Nursing Management, vol. 11, no. 3, pp. 177-188, May 2003.

[14] S. Cruz, "A supervisão clínica em enfermagem como estratégia de qualidade no contexto da enfermagem avançada," Revista Servir, vol. 56 , no. 5, pp. 112-118, 2008.

[15] K. Hyrkäs et al., "The effects of clinical supervision on the quality of care: examining the results of team supervision," Journal of Advanced Nursing, vol. 33, no. 4, pp. 492-502, Feb. 2001.

[16] J. Lee and S. Miller, "A self-care framework for social workers: Building a strong foundation for practice," Families in Society, vol. 94, no. 2, pp. 96-103, Sep. 2013.

[17] I. Slusher et al., "Appalachian women: Health beliefs, self-care, and basic conditioning factors," Journal Cultural Diversity, vol. 17, no. 3, pp. 84-89, 2010.
[18] A. Wilkinson and L. Whitehead, "Evolution of the concept of self-care and implications for nurses: A literature review," International Journal Nursing Studies, vol. 46, no. 8, pp. 1143-1147, Aug. 2009.

[19] S. Cruz and A. Carvalho, "Portuguese version of the Manchester Clinical Supervision Scale: the translation and validation process," Journal of Educational Sciences \& Psychology, vol. 2, no. 1, pp. 123, 2012.

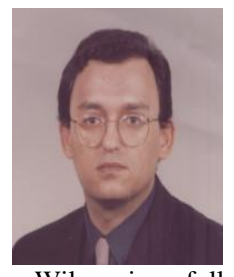

Wilson Correia de Abreu earned his Ph.D. in education with an emphasis in Adult Education at the University of Lisbon in 1999 and the master in education Sciences in 1994, at the same University. He earned also the aggregation in health sciences in 2006 at the University of Aveiro. Wilson is a senior member of the International Economics Development Research Center (IEDRC).

Wilson is a full professor at the Porto School of Nursing, where he coordinates the Master Course on Mental Health and Psychiatry. In the last years, he has collaborated with Universities and other organizations, teaching anthropology, clinical supervision, psychiatry and qualitative methods. International experiences (research and teacher mobility) included the majority of the European Countries and Brazil, USA, Canada, Turkey and Russia.

During his professional life, Wilson presented more than 150 conferences in Portugal and abroad and was a member of European Projects (European Networks, Leonardo da Vinci, Grundvig). He collaborates with the University of Porto, Lisbon, Aveiro, Azores and Evora advising and examining $\mathrm{PhD}$ thesis. Three of his books are known worldwide ("Health, illness and cultural diversity" (Lisbon: Editora Piaget: 2003, 2013 - 2 editions), "Transitions in multicultural contexts" (Coimbra, Formasau: 2008, 2011 - 2 editions) and "Education and learning in clinical context" (Coimbra, Formasau: 2007). The author's major fields of studies and research interests include mental health and psychiatric disorders (dementia, depression, and suicide), clinical education, clinical supervision and anthropology of health. Prof. Abreu is actually the President of the Board of Curators of the Polytechnic Institute of Portalegre, a member of the Scientific Commission for Doctoral Studies in Nursing (University of Lisbon), a collaborator of the Agency for Assessment and Accreditation of Higher Education (A3ES) (Portugal) and also a member of ASMELP (Mental Health and Psychiatric Association of the Portuguese Speaking Countries).

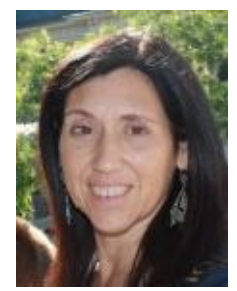

Cristina Barroso Pinto took her PhD in didactics at the University of Aveiro in 2011 and the master in bioethics in 2001, at the University of Porto. The major fields of study are bioethics, medical-surgical nursing, clinical supervision and qualitative methods. She is an adjoint professor at the Porto School of Nursing since 1999. In the last years, she has collaborated with another Nursing School (Instituto Piaget). She teaches bioethics and medical-surgical nursing. International experiences (teacher mobility) included some European Countries

During her professional life, Prof. Pinto presented a set of conferences in Portugal and abroad and has experiences on European Projects (Leonardo da Vinci).

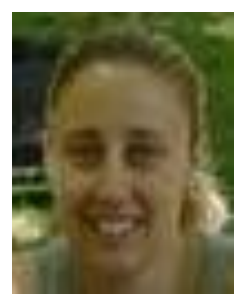

Maria de Fátima Moreira is a $\mathrm{PhD}$ student in the University of Aveiro. She earned her master in public health in 2004 at the Faculty of Medicine of the University of Porto.

Fátima Moreira is an adjoint professor at the Porto School of Nursing, where she teaches medical-surgical nursing. International experiences (teacher mobility) included some European Countries.

The author's major field of studies and research interests includes clinica education and clinical supervision.

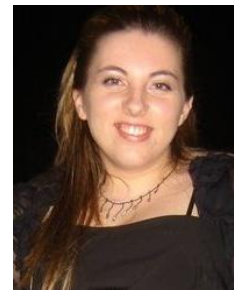

Sílvia Marlene Teixeira earned her master in clinical supervision in nursing at Porto School of Nursing in 2012.

Sílvia is a nurse at "Obra Diocesana de Promoção Social" in Porto where she coordinates and supervises a group of carers. She also works as a nurse in a nursing home and develop nursing care in the community. During her professional life, Sílvia presented some conferences in Portugal related to 